\title{
Combination of afatinib with cetuximab in patients with EGFR-mutant non-small-cell lung cancer resistant to EGFR inhibitors
}

This article was published in the following Dove Press journal:

OncoTargets and Therapy

19 May 2015

Number of times this article has been viewed

\author{
Jéssica Ribeiro Gomes \\ Marcelo Rocha S Cruz \\ Antonio Ermirio de Moraes Oncology \\ Center, São Paulo-Brazil
}

\begin{abstract}
Tyrosine kinase inhibitors (TKIs) targeting the epidermal growth factor receptor (EGFR) have shown effectiveness for advanced non-small-cell lung cancer (NSCLC) with activating mutations in the EGFR gene. However, resistance to the EGFR TKIs develops mostly secondary to T790M mutation in exon 20 . The use of afatinib associated with cetuximab represents a new possibility of therapy following progression on gefitinib or erlotinib. We present two patients who acquired resistance to first-generation TKI and who underwent combination treatment with afatinib plus cetuximab as third-line therapy. Both patients presented partial response, and the time duration of disease control was 8 months and 10 months. The combined use of afatinib plus cetuximab emerges as a new possibility for the treatment of patients with advanced NSCLC harboring mutated EGFR after progression on first-generation EGFR TKIs with consequently acquired resistance to TKIs. Further studies are necessary to consolidate the data.
\end{abstract}

Keywords: lung cancer, non-small-cell lung cancer, EGFR, afatinib, cetuximab, case report

\section{Introduction}

Lung cancer represents the major cause of cancer-related mortality in the US. The estimated number of deaths due to lung cancer in 2014 is 159,260, with an expected 5-year survival of 16.6\%. ${ }^{1,2}$ Tyrosine kinase inhibitors (TKIs) targeting the epidermal growth factor receptor (EGFR) have shown effectiveness as therapy for advanced nonsmall-cell lung cancer (NSCLC) with activating mutations in the EGFR gene, especially in the exons 19 and 21..$^{3-6}$ The first-generation EGFR TKIs, erlotinib and gefitinib, are associated with response rates of approximately $60 \%-70 \%$ when administered to those harboring activating mutations in EGFR, with superior progression-free survival (PFS) when compared to chemotherapy..$^{3-8}$

However, resistance will eventually ensue to the EGFR TKIs, with consequent disease progression. Different mechanisms of resistance to TKIs have been described, such as secondary mutations in the EGFR gene, amplification of Human Epidermal Growth Factor Receptor 2 (HER2) gene, mutations in PIK3CA and BRAF, and conversion to small-cell lung cancer. ${ }^{9}$ The most important mechanism of resistance is a secondary mutation in the EGFR gene, the T790M mutation in exon 20, responsible for about $50 \%$ of cases. ${ }^{10}$

Afatinib, a second-generation TKI, acts as an irreversible ErbB family blocker (including EGFR and HER2), and has shown activity as single agent in EGFR-mutant, TKI-naïve patients. The Phase III trial LUX-Lung 3 yielded an increase in median PFS in patients with mutated EGFR treated with afatinib when compared with cisplatin and pemetrexed as first-line therapy: 11.1 months and 6.9 months, respectively (hazard
Correspondence: Marcelo Rocha S Cruz Rua Martiniano de Carvalho, 95I Bela Vista, São Paulo-SP, Brazil CEP 0I32I-00।

Email marcelo.cruz.md@gmail.com 
ratio $[\mathrm{HR}] 0.58 ; 95 \%$ confidence interval $[\mathrm{CI}]: 0.43-0.78$, $P=0.001){ }^{11}$

At the 2014 American Society of Clinical Oncology meeting, a pooled analysis of two randomized Phase III trials, LUX-Lung 3 and LUX-Lung 6, was presented. The latter compared first-line afatinib with the combination of gemcitabine and cisplatin in EGFR-mutant patients. For the first time, results showed an increase in overall survival (OS) with afatinib in the group of mutated EGFR patients with deletion in exon 19. The median OS was of 27.3 months for the afatinib group and 24.3 months for the chemotherapy group (HR $0.81, P=0.037$ ). Among patients with deletion in exon 19 , the HR was 0.59 , CI 0.45 , and $P<0.001$. An update of individual analysis of LUX-Lung 3 and LUX-Lung 6 also yielded increased OS in patients with deletion in exon 19 (HR 0.54, $P=0.0015$ and HR 0.64, $P=0.0229$, respectively). ${ }^{12}$

Newer EGFR TKIs are also under development, with promising results. The compounds CO-1686 and AZD9291 have recently showed clinical response in patients with EGFR-mutant NSCLC previously exposed to first-generation TKIs and with acquired T790M mutation. ${ }^{13,14}$ Moreover, the combination of afatinib and cetuximab, an anti-EGFR antibody, showed impressive response rate and disease control in patients with mutated EGFR NSCLC that progressed after erlotinib or gefitinib. ${ }^{15,16}$ Here, we present two cases that evaluate the treatment with the combination of afatinib plus cetuximab after progression on platinum-based chemotherapy and first-generation TKI in patients with EGFR-mutant advanced NSCLC.

\section{Case reports}

\section{Patient I}

A 54-year-old white male, nonsmoker, presented with dry cough in September 2009. A computed tomography (CT) scan of the chest showed nodules scattered in the right lung. No alteration was detected in the CT of abdomen/pelvis, magnetic resonance imaging of the brain, or bone scintigraphy. A biopsy of the lung nodule revealed an adenocarcinoma. Mutational analysis of EGFR showed a rare exon 18 mutation, which was available 4 weeks later. ${ }^{17}$ Due to this delay, the patient underwent first-line chemotherapy with carboplatin AUC 6 intravenous (IV) D1, pemetrexed $500 \mathrm{mg} / \mathrm{m}^{2}$ IV D1, and bevacizumab $15 \mathrm{mg} / \mathrm{kg}$ IV D1 every 3 weeks for four cycles, until December 2009, with partial response. In January 2010, erlotinib was introduced at a dose of $150 \mathrm{mg}$ per day, with initial partial response. A grade 2 rash was observed during the first 2 weeks of treatment, evolving to grade 4 rash even after recommended supportive therapies.
Thus, erlotinib was discontinued for 1 week and reintroduced at a lower dose of $100 \mathrm{mg}$ PO daily. The patient had stable disease with this regimen until January 2012, when he presented with pain in the right hemithorax. A positron emission tomography-computed tomography scan detected disease progression in the lung, bone, and mediastinal lymph nodes. The dose of erlotinib was increased to $150 \mathrm{mg}$ per day, and zoledronic acid $4 \mathrm{mg}$ monthly was introduced. The patient remained with stable disease until July 2012, when a new lesion in T3 was noted representing disease progression. Stereotactic radiosurgery was performed in this vertebra with a 16 Gy single dose.

In October 2012, new symptomatic lesions in the acetabulum and right femur appeared along with progression of disease in the lung and lymph nodes. Anti-algic radiotherapy of new and symptomatic bone lesions was administered. Moreover, erlotinib was suspended, and systemic therapy with cetuximab $500 \mathrm{mg} / \mathrm{m}^{2} \mathrm{IV}$ D1 every 2 weeks associated with afatinib $40 \mathrm{mg}$ PO daily was initiated. There was a major partial response in the lung, lymph nodes, and bone lesions, with subsequent stable disease and clinical benefit for 10 months of treatment (Figure 1). After 3 months, the patient presented with a grade 3 diffuse rash with pustule lesions mainly at the trunk, dorsum, and legs, which was treated with antibiotic and oral corticosteroid, with resolution. The afatinib dose was reduced to $30 \mathrm{mg}$ per day.

Ten months after treatment initiation, the patient presented with a right brachial and crural paresis and echolalia. Brain magnetic resonance imaging showed multiple small nodules consistent with brain metastases. Afatinib and cetuximab were suspended, and whole brain radiation therapy was performed in September 2013. After radiotherapy, patient was submitted to systemic treatment with docetaxel and erlotinib regimen.

\section{Patient 2}

A 67-year-old white male, former smoker, with a prior history of atrial fibrillation and arterial hypertension presented with hemoptysis in 2009. In September 2009, a CT scan of the chest showed a solid lesion in the medial region of the left lung, associated with ipsilateral pleural effusion. The CT of the abdomen and pelvis showed two hepatic nodules, but there was no evidence of neoplastic disease detected at biopsy. Biopsy of the lung lesion revealed adenocarcinoma, with wild-type EGFR.

The patient underwent systemic therapy with cisplatin $50 \mathrm{mg} / \mathrm{m}^{2}$ IV D1, D8, D29, and D36 plus etoposide $50 \mathrm{mg} / \mathrm{m}^{2}$ IV D1-D5 and D29-D33 for two cycles, followed by thoracic radiotherapy (61 Gy) plus chemotherapy for two 

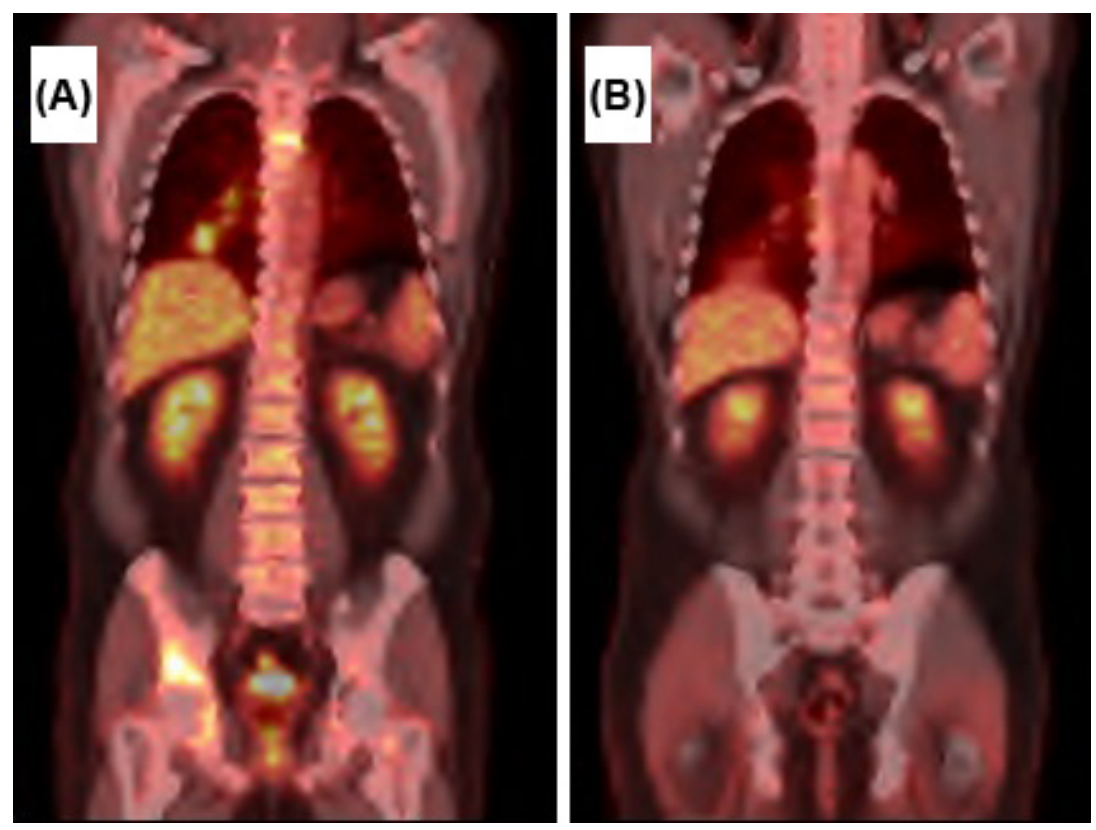

Figure I PET-CT scan of patient I.

Notes: (A) Baseline for afatinib and cetuximab therapy. (B) After 6 months of afatinib and cetuximab therapy, with major partial response. Abbreviation: PET-CT, positron emission tomography-computed tomography.

more cycles, until January 2010. Maintenance therapy with pemetrexed was performed, and the patient was submitted to pleurodesis in April 2010. After 18 cycles of pemetrexed, the disease progressed with symptomatic metastases detected in the thoracic spine and left iliac bone. Erlotinib $150 \mathrm{mg}$ per day was introduced, associated with zoledronic acid and local radiotherapy. The patient showed benefit with erlotinib, which was maintained for about 16 months when hepatic metastases were diagnosed.

Because of the prolonged benefit associated with the use of erlotinib, a liver metastasis biopsy was performed, which detected an EGFR mutation: deletion in exon 19 and T790M mutation in exon 20. He was started on chemotherapy (carboplatin AUC 6 IV D1 plus paclitaxel $200 \mathrm{mg} / \mathrm{m}^{2}$ IV D1 plus bevacizumab $15 \mathrm{mg} / \mathrm{kg}$ IV D1 every 3 weeks) for six cycles, combined with erlotinib, from October 2012 to January 2013, with significant partial response. Maintenance therapy with bevacizumab and erlotinib was performed with stable disease for 3 months, until disease progression in May 2013 in the lung, bone, and liver. Bevacizumab and erlotinib were discontinued, and afatinib $40 \mathrm{mg}$ PO daily combined with cetuximab $500 \mathrm{mg} / \mathrm{m}^{2} \mathrm{IV}$ D1 every 2 weeks was initiated.

The EGFR combined therapy yielded partial response. The patient presented with grade 3 cutaneous acneiform lesions, and so antibiotics and corticosteroids were introduced. The cetuximab dose interval was therefore increased to every 3 weeks. However, due to persistent acneiform lesions, and development of ungueal lesions, there was a further need to increase the dosing interval to every 4 weeks.

After initial partial response, stable disease was achieved, with use of afatinib plus cetuximab for 8 months, until hepatic disease progression in January 2014. The treatment was then substituted for navelbine plus gemcitabine, which the patient is currently undertaking.

\section{Discussion}

The first-generation EGFR TKIs gefitinib and erlotinib are associated with significant response rates as high as $70 \%$ in advanced NSCLC with activating EGFR mutation. ${ }^{3-8}$ However, resistance eventually ensues secondary to T790M mutation in the exon 20 , or less frequently, to the amplification of the MET proto-oncogene. ${ }^{9,10}$

Approximately $50 \%$ of patients with mutated EGFR who develop acquired resistance to EGFR TKIs have a second-site mutation T790M in the threonine gatekeeper residue besides the primary EGFR-activating mutation. The consequent resistance to EGFR TKI is possibly due to altered drug binding in the ATP pocket of EGFR, or to increased affinity of the EGFR-L858R for ATP, reducing the potency of an ATP-competitive kinase inhibitor. ${ }^{9}$ There are other secondsite mutations in EGFR also associated with acquired TKI resistance: T854A (exon 21), L747S (exon 19), and D761Y (exon 19). ${ }^{18-20}$ MET amplification can be observed in about $20 \%$ of NSCLCs with acquired resistance to EGFR TKI. The resistance occurs through activation of MET-PI3K signaling 
and of AKT through ErbB3-mediated activation of PI3K in the presence of EGFR TKIs. ${ }^{21}$ Other mechanisms related to acquired resistance to TKI currently being investigated include mutations in PIK3CA, epithelial-to-mesenchymal transition, histological transformation from NSCLC into small-cell lung cancer, activation of AXL receptor tyrosine kinase through overexpression or upregulation of its ligand GAS6, loss of inhibitory IGF1R and loss or deduction of PTEN pathways, amplification of HER2 genes, and mutations in BRAF. ${ }^{9}$

In patients with disease progression secondary to acquired resistance to erlotinib or gefitinib, the possibility of incorporating other targeted therapies has been emerging with agents such as afatinib and cetuximab, further delaying chemotherapy use. Moreover, the response rate to third and further lines of chemotherapy is below 5\%, with median PFS not superior to 2 months.

An alternative strategy also explored is the use of different dosing schedules of EGFR TKIs, such as the one performed in case 1 , who was maintained on erlotinib at a lower dose (100 mg per day), achieving disease control for several months by increasing the dose to $150 \mathrm{mg}$ per day at disease progression. ${ }^{22}$

Afatinib, a second-generation TKI, acts by blocking irreversibly the EGFR and other members of the ErbB family of receptors. Preclinical studies showed that afatinib could be a potent inhibitor of cells with T790M mutation, making it possible to overcome T790M-driven acquired resistance. ${ }^{23}$ However, the use of afatinib after progression on gefitinib or erlotinib was evaluated in the LUX-Lung 1 trial, which randomized patients to placebo or afatinib following progression on a first-generation TKI. The response rate was only $7 \%$ in patients treated with afatinib, and the median PFS was of 3.3 months and 1.1 months in the afatinib and placebo group, respectively (HR $0.38, P<0.0001$ ), with no significant difference in OS. ${ }^{24}$

Cetuximab, a monoclonal antibody that binds competitively with high affinity to EGFR, has also been studied following TKI progression, with poor activity detected as single agent. In a small trial, the response rate was $0 \%$, with $28 \%$ stable disease. Median PFS was of 1.8 months, and OS was of 7.5 months. ${ }^{25}$ However, the combination of afatinib and cetuximab could result in better outcomes after progression on first-generation TKI in advanced NSCLC. Although neither single-agent afatinib nor single-agent cetuximab has significant activity in EGFR TKI-resistant NSCLC, their combination has been proposed to have activity following progression on gefitinib or erlotinib. The benefit could be due to the dual inhibition of EGFR, lowering the EGFR pathway signaling, even when there is a resistance mutation. In preclinical studies, there was disease regression with afatinib plus cetuximab, whereas the use of both agents alone presented no activity. ${ }^{26}$

A Phase II study evaluated the combined treatment (afatinib plus cetuximab) and showed disease control in all 26 patients with clinically defined acquired resistance, detecting partial response in 36\%, including patients with T790M mutation. ${ }^{27}$ An update of this trial evaluated the combined use of afatinib $40 \mathrm{mg}$ oral daily with cetuximab $500 \mathrm{mg} / \mathrm{m}^{2}$ every 2 weeks in 100 patients with mutated EGFR advanced NSCLC, who progressed on erlotinib or gefitinib. The rate of disease control was of $94 \%$, with objective response observed in $40 \%$ in the first 60 patients evaluated. The PFS in 9 months was of $18 \%$. Median PFS was of 4.7 months, and the median duration of response was 7.7 months. Responses occurred in patients with or without T790M mutations. ${ }^{15}$ Recent publications, which included more patients, reported median PFS of 4.7 months and median duration of response of 5.7 months. ${ }^{16}$

Toxicity is also a significant concern with the dual EGFR inhibition, because of the potential for overlapping side effects. In the study, the most common adverse events included rash (90\%), diarrhea (71\%), nail effects (57\%), and stomatitis (56\%). The grade 3 events were of $20 \%, 6 \%, 0 \%$, and $1 \%$, respectively. Less common side effects were fatigue, nausea, xerosis, pruritus, and headache. ${ }^{16}$ Thus, close evaluation of these patients is required with early intervention for toxicities, which may include dose delays or reductions.

In our two cases herein presented, the duration of disease control were favorable at 8 months and 10 months, considering that this PFS was seen in only a minority of patients (18\%) in the Phase II study by Janjigian et al. ${ }^{15}$ Both patients presented partial response even though afatinib plus cetuximab was a third-line therapy.

With respect to toxicity, both patients presented dermatologic lesions, requiring antibiotics, corticosteroids, and dose reductions (of afatinib or cetuximab).

Our results confirm that, in daily clinical practice, some patients may benefit from afatinib combined with cetuximab after progression on TKI, where the ErbB signal still has importance in oncogenic pathways. Therefore, the results are encouraging, creating a new possibility for targeted therapy in advanced NSCLC patients with mutated EGFR who acquired resistance to first-generation TKIs. However, 
more data are necessary in order to confirm the results and to better access the toxicity profile. In the future, other questions may arise, such as whether the combination therapy with afatinib plus cetuximab could be used after progression on second-generation TKIs, and even whether it would be more effective as first-line therapy compared to TKIs alone.

\section{Conclusion}

The combining use of afatinib plus cetuximab emerges as a new possibility for the treatment of patients with advanced NSCLC harboring mutated EGFR after progression on first-generation EGFR TKIs (erlotinib or gefitinib), with consequently acquired resistance to TKIs. The initial studies showed activity in this scenario, as in the two case reports presented in this article, probably indicating that these NSCLC patients still depend on ErbB signaling for the development of cancer progression. Further studies are necessary to consolidate the data.

\section{Acknowledgments}

The authors would like to thank the patients reported in the article, as well as Phillip Scheinberg, MD for being the text revisor.

\section{Disclosure}

Jéssica Ribeiro Gomes has no potential conflicts of interest. Marcelo Rocha de Souza Cruz is a medical advisor of Boehringer Ingelheim ${ }^{\circledR}$ Advisory Board. The author reports no other conflict of interest in this work.

\section{References}

1. Surveillance, Epidemiology, and End Results Program (SEER) Database. 2014. Available from: http://seer.cancer.gov/statfacts/html/lungb.html. Accessed January 1, 2014.

2. National Comprehensive Cancer Network (NCCN). 2014. Available from: http://www.nccn.org/. Accessed January 1, 2014.

3. Fukuoka M, Wu YL, Thongprasert S, et al. Biomarker analyses and final overall survival results from a phase III, randomized, open-label, firstline study of gefitinib versus carboplatin/paclitaxel in clinically selected patients with advanced non-small-cell lung cancer in Asia (IPASS). J Clin Oncol. 2011;29(21):2866-2874.

4. Rosell R, Carcereny E, Gervais R, et al; Spanish Lung Cancer Group in collaboration with Groupe Français de Pneumo-Cancérologie and Associazione Italiana Oncologia Toracica. Erlotinib versus standard chemotherapy as first-line treatment for European patients with advanced EGFR mutation-positive non-small-cell lung cancer (EURTAC): a multicentre, open-label, randomised phase 3 trial. Lancet Oncol. 2012;3(3):239-246.

5. Mok TS, Wu YL, Thongprasert S, et al. Gefitinib or carboplatin-paclitaxel in pulmonary adenocarcinoma. $N$ Engl J Med. 2009;361(10):947-957.

6. Zhou C, Wu YL, Chen G, et al. Erlotinib versus chemotherapy as first-line treatment for patients with advanced EGFR mutation-positive non-smallcell lung cancer (OPTIMAL, CTONG-0802): a multicentre, open-label, randomised, phase 3 study. Lancet Oncol. 2011;2(8):735-742.
7. Maemondo M, Inoue A, Kobayashi K, et al; North-East Japan Study Group. Gefitinib or chemotherapy for non-small-cell lung cancer with mutated EGFR. N Engl J Med. 2010;362(25):2380-2388.

8. Han JY, Park K, Kim SW, et al. First-SIGNAL: first-line single-agent iressa versus gemcitabine and cisplatin trial in never-smokers with adenocarcinoma of the lung. J Clin Oncol. 2012;30(10):1122-1128.

9. Lin L, Bivona TG. Mechanisms of resistance to epidermal growth Factor receptor inhibitors and novel therapeutic strategies to overcome resistance in NSCLC patients. Chemother Res Pract. 2012;2012:817297.

10. Ma C, Wei S, Song Y. T790M and acquired resistance of EGFR TKI: a literature review of clinical reports. J Thorac Dis. 2011;3(1):10-18.

11. Sequist LV, Yang JC, Yamamoto N, et al. Phase III study of afatinib or cisplatin plus pemetrexed in patients with metastatic lung adenocarcinoma with EGFR mutations. J Clin Oncol. 2013;31(27):3327-3334.

12. Yang JC, Sequist LV, Schuler MH, et al. Overall survival (OS) in patients (pts) with advanced non-small cell lung cancer (NSCLC) harboring common (Del19/L858R) epidermal growth factor receptor mutations (EGFR mut): pooled analysis of two large open-label phase III studies (LUXLung 3 [LL3] and LUX-Lung 6 [LL6]) comparing afatinib with chemotherapy (CT). J Clin Oncol. 2014;32:5s. [suppl; abstr 8004].

13. Sequist LV, Soria J, Gadgeel SM, et al. First-in-human evaluation of CO-1686, an irreversible, highly selective tyrosine kinase inhibitor of mutations of EGFR (activating and T790M). J Clin Oncol. 2014;32:5s. [suppl; abstr 8010].

14. Janne PA, Ramalingam SS, Yang JC, et al. Clinical activity of the mutant-selective EGFR inhibitor AZD9291 in atients (pts) with EGFR inhibitor - resistant non-small cell lung cancer (NSCLC). J Clin Oncol. 2014;32:5s. [suppl; abstr 8009].

15. Janjigian YY, Smit EF, Horn L, et al. Activity of afatinib/cetuximab in patients (pts) with EGFR mutant non-small cell lung cancer (NSCLC) and acquired resistance (AR) to EGFR inhibitors. Ann Oncol. 2012;23. [supp19; abstr 12270].

16. Janjigian YY, Smit EF, Groen HJM, et al. Dual inhibition of EGFR with afatinib and cetuximab in kinase inhibitor-resistant $E G F R$-mutant lung cancer with and without T790M mutations. Cancer Discov. 2014;4(9):1036-1045.

17. Brandão EP, Pantarotto MG, Cruz M. A novel EGFR mutation in exon 18 with high sensitivity to EGFR TKI treatment with reduced dose. J Thoracic Oncol. 2012;7(11):e32.

18. Bean J, Riely GJ, Balak M, et al. Acquired resistance to epidermal growth factor receptor kinase inhibitors associated with a novel T854A mutation in a patient with EGFR-mutant lung adenocarcinoma. Clin Cancer Res. 2008;14(2):7519-7525.

19. Costa DB, Schumer ST, Tenen SG, Kobayashi S. Differential responses to erlotinib in epidermal growth factor receptor (EGFR)-mutated lung cancers with acquired resistance to gefitinib carrying the L747S or T790M secondary mutations. J Clin Oncol. 2008;26(7):1182-1184.

20. Balak MN, Gong Y, Riely GJ, et al. Novel D761Y and common secondary T790M mutations in epidermal growth factor receptor-mutant lung adenocarcinomas with acquired resistance to kinase inhibitors. Clin Cancer Res. 2006;12(21):6494-6501.

21. Engelman JA, Zejnullahu K, Mitsudomi T, et al. MET amplification leads to gefitinib resistance in lung cancer by activating ERBB3 signaling. Science. 2007;316(5827):1039-1043.

22. Chmielecki J, Foo J, Oxnard GR, et al. Optimization of dosing for EGFR-mutant non-small cell lung cancer with evolutionary cancer modeling. Sci Transl Med. 2011;3(90):90ra59.

23. Li D, Ambrogio L, Shimamura T, et al. BIBW2992, an irreversible EGFR/HER2 inhibitor highly effective in preclinical lung cancer models. Oncogene. 2008;27(34):4702-4711.

24. Miller VA, Hirsh V, Cadranel J, et al. Afatinib versus placebo for patients with advanced, metastatic non-small-cell lung cancer after failure of erlotinib, gefitinib, or both, and one or two lines of chemotherapy (LUX-Lung 1): a phase 2b/3 randomised trial. Lancet Oncol. 2012;13(5):5239-5285. 
25. Neal JW, Heist RS, Fidias P, et al. Cetuximab monotherapy in patients with advanced nonsmall cell lung cancer after prior epidermal growth factor receptor tyrosine kinase inhibitor therapy. $J$ Thorac Oncol. 2010;5(11):1855-1858.

26. Regales L, Gong Y, Shen R, et al. Dual targeting of EGFR can overcome a major drug resistance mutation in mouse models of EGFR mutant lung cancer. J Clin Invest. 2009;119(10):3000-3010.
27. Janjigian YY, Groen HJ, Horn L, et al. Activity and tolerability of afatinib (BIBW 2992) and cetuximab in NSCLC patients with acquired resistance to erlotinib or gefitinib. J Clin Oncol. 2011;29:149. [suppl; abstr 7525].

\section{Publish your work in this journal}

OncoTargets and Therapy is an international, peer-reviewed, open access journal focusing on the pathological basis of all cancers, potential targets for therapy and treatment protocols employed to improve the management of cancer patients. The journal also focuses on the impact of management programs and new therapeutic agents and protocols on

\section{Dovepress}

patient perspectives such as quality of life, adherence and satisfaction. The manuscript management system is completely online and includes a very quick and fair peer-review system, which is all easy to use. Visit http://www.dovepress.com/testimonials.php to read real quotes from published authors.

Submit your manuscript here: http://www.dovepress.com/oncotargets-and-therapy-journal 